\title{
Persistent URLs and Citations Offered for Digital Objects by Digital Libraries
}

Nicholas Homenda

\begin{abstract}
As libraries, archives, and museums make unique digital collections openly available via digital library platforms, they expose these resources to users who may wish to cite them. Often several URLs are available for a single digital object, depending on which route a user took to find it, but the chosen citation URL should be the one most likely to persist over time. Catalyzed by recent digital collections migration initiatives at Indiana University Libraries, this study investigates the prevalence of persistent URLs for digital objects at peer institutions and examines the ways their platforms instruct users to cite them. This study reviewed institutional websites from the Digital Library Federation's (DLF) published list of 195 members and identified representative digital objects from unique digital collections navigable from each institution's main web page in order to determine persistent URL formats and citation options.
\end{abstract}

Findings indicate an equal split between offering and not offering discernible persistent URLs with four major methods used: Handle, DOI, ARK, and PURL. Significant variation in labeling persistent URLs and inclusion in item-specific citations uncovered areas where the user experience could be improved for more reliable citation of these unique resources.

\section{INTRODUCTION}

Libraries, archives, and museums often make their unique digital collections openly available in digital library services and in different contexts, such as digital library aggregators like the Digital Public Library of America (DPLA, https://dp.la/) and HathiTrust Digital Library (https://www.hathitrust.org/). As a result, there can be many URLs available that point to digital objects within these collections. Take, for example, Image Collections Online (http://dlib.indiana.edu/collections/images) at Indiana University (IU), a service launched in 2007 featuring open access IU image collections. Users discover images on the site through searching and browsing and its collections are also shared with DPLA. The following URLs exist for the digital object shown in figure 1, an image from the Building a Nation: Indiana Limestone Photograph Collection:

- The URL as it appears in the browser in Image Collections Online: https://webapp1.dlib.indiana.edu/images/item.htm?id=http://purl.dlib.indiana.edu/iudl/i mages/VAC5094/VAC5094-01446

- The Persistent URL on that page ("Bookmark this page at") http://purl.dlib.indiana.edu/iudl/images/VAC5094/VAC5094-01446

- The URL pasted from the browser for the image in DPLA: https://dp.la/item/eb83ff0a6ae507e2ba441634f7eb0f18?q=indiana\%20limestone

Nicholas Homenda (nhomenda@indiana.edu) is Digital Initiatives Librarian, Indiana University Bloomington. (C) 2021. 
As a digital library or collection manager, which URL would you prefer to see cited for this object?

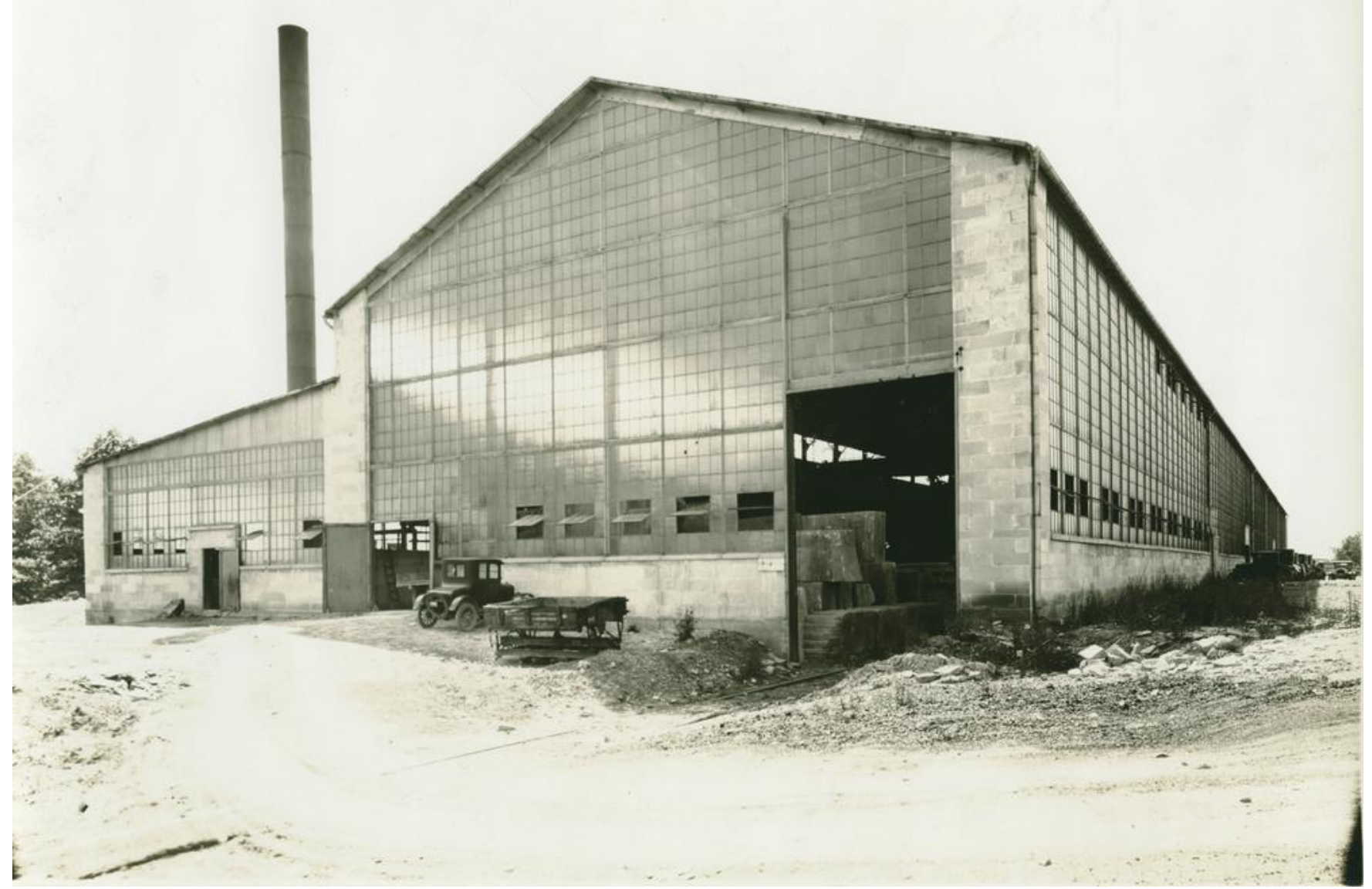

Figure 1. An example of a digital object with multiple URLs. McMillan Mill, ILCO ID IN2288 1. Courtesy, Indiana Geological and Water Survey, Indiana University, Bloomington, Indiana. Retrieved from Image Collections Online at http://purl.dlib.indiana.edu/iudl/images/VAC5094/VAC5094$\underline{01446 .}$

Citation instructions given to authors in major style guides explicitly mention using the best possible form of a resource's URL: "[I]t is important to choose the version of the URL that is most likely to continue to point to the source cited."1 Of the three URLs above, the second is a PURL, or Persistent URL (https://archive.org/services/purl/), which is why both Image Collections Online and DPLA instruct users to bookmark or cite it. Other common methods for issuing and maintaining persistent URLs include Digital Object Identifiers (DOI, https://www.doi.org/), Handles (http://handle.net/), and Archival Resource Keys (ARK, https://n2t.net/e/ark ids.html). All of those have been around since the late 1990s to early 2000s.

At Indiana University Libraries, recent efforts have focused on migrating digital collections to new digital library platforms, mainly based on the open source Samvera repository software (https://samvera.org/). As part of these efforts, we wanted to survey how peer institutions were 
employing persistent, citable URLs for digital objects to determine if a prevailing approach had emerged since Indiana University Libraries' previous generation of digital library services were developed in the early- to mid-2000s. Besides having the capability of creating and reliably serving these URLs, our digital library platforms need to make these URLs easily accessible to users, preferably along with some assertion that the URLs should be used when citing digital objects and collections instead of the many non-persistent URLs also directing to those same digital objects and collections.

Although libraries, archives, and museums have digitized and made digital objects in digital collections openly accessible for decades using several methods for providing persistent, citable URLs, how do institutions now present digital object URLs to people who encounter, use, and cite them? By examining digital collections within a large population of digital library institutions' websites, this study aims to discover

1. What methods of URL persistence are being employed for digital objects by digital library institutions?

2. How do these institutions' websites instruct users to cite these digital objects?

\section{LITERATURE REVIEW}

The study of digital objects in the literature often takes a philosophical perspective in attempting to define them. Moreover, practical accounts of digital object use and reuse note the challenges associated with infrastructure, retrieval, and provenance. Much of the literature about common methods of persistent URL resolution comes from individuals and entities who developed and maintain these standards, as well as overviews of the persistent URL resolution methods available. Finally, several studies have investigated the problem of "link rot" by tracking the availability of web-hosted resources over time.

Allison notes the generations of philosophical thought that it took to recognize common characteristics of physical objects and the difficulty in understanding an authentic version of a digital object, especially with different computer hardware and software changing the way digital objects appear. ${ }^{2}$ Hui also investigates the philosophical history of physical objects to begin to define digital objects through his methods of datafication of objects and objectification of data, noting that digital objects can be approached in three phases: objects, data, and networks, in order to define them. ${ }^{3}$ Lynch is also concerned with determining the authenticity of digital objects and challenges inherent in the digital realm. In describing digital objects, he creates a hierarchy with raw data at the bottom, elevated to interactive experiential works at the top which elicit the fullest emotional connection contributing to the authentic experience of the work. ${ }^{4}$

The literature often examines digital objects from the practitioner's perspective, such as the publishing industry's difficulty in repurposing digital objects for new publishing products. Publishers in Benoit and Hussey's 2011 case study note the tension between managers and technical staff concerning assumptions about what their computer system could automatically do with their digital objects; their digital objects always require some human labor and intervention to be accurately described and retrievable later. ${ }^{5}$ Dappert et al. note the need to describe a digital object's environment in order to be able to reproduce it in their work with the PREMIS Data Dictionary for Preservation Metadata (https://www.loc.gov/standards/premis/). ${ }^{6}$ Strubulis et al. provide a model for digital object provenance using inference and Resource Description Framework (RDF) triples (https://w3.org/RDF/) since storing full provenance information for 
complex digital objects, such as the large amount of Mars rover data they offer as an example, would be cost prohibitive. ${ }^{7}$

In 2001, Arms describes the landscape of persistent Uniform Resource Names (URN) of Handles, PURLs, and DOIs near the latter's inception. ${ }^{8}$ Recent work by Koster explains the persistent identifier methods most in use today and examines current infrastructure practices for maintaining them. ${ }^{9}$ The persistent link resolution method most prominently featured in the literature is the Digital Object Identifier (DOI). Beginning in 1999, those behind developing and implementing DOI have explained its inception, development, and trajectory, continuing with Paskin's deep explanation in 2002 of the reasons why DOI exist and the technology behind the service. ${ }^{10}$ Discipline-specific research notes the utility of DOI. Sidman and Davidson and Weissberg studied DOI for the purposes of automating the supply chain in the publishing industry. ${ }^{11}$ DeRisi, Kennison, and Twyman, on behalf of the Public Library of Science (PLoS) announced their 2003 decision to broadly implement DOI, followed by additional disciplinespecific encouragement of the practice by Skiba in nursing education and Neumann and Brase in molecular design. ${ }^{12}$

The Archival Resource Key (ARK) is an alternative permanent link resolution scheme. Since 2001, the open-source ARK identifier offers a self-hosted solution for providing persistent access to digital objects, their metadata, and a maintenance commitment. ${ }^{13}$ Recently, Duraspace working groups have planned for further development and expansion of ARK with the ARKs in the Open Project (https://wiki.lyrasis.org/display/ARKs/ARKs+in+the+Open+Project).

Persistent URLs (PURLs) have been used to provide persistent access to digital objects for nearly 20 years, and their use in the library community is well documented. Shafer, Weibel, and Jul anticipate Uniform Resource Names becoming a Web standard and offer PURLs as an intermediate step to aid in URN development. ${ }^{14}$ Shafer also explained how OCLC uses PURLs and Alternate Routing Methods (ARMs) to properly direct global users to OCLC resources. ${ }^{15}$ PURLs are also used to provide persistent access to government information and were seen by the Cendi Persistent Identification Task Group as essential to their early efforts to implement the Federal Enterprise Architecture (FEA) and a theoretical Federal Persistent Identification Resolver. ${ }^{16}$

Digital objects and collections should ideally be accessible via URLs that work beyond the life of any one platform, lest the materials be subjected to "link rot," or the process of decay when previously working links no longer correctly resolve. Ducut et al. investigated 1994-2006 MEDLINE abstracts for the presence of persistent link resolution services such as Handle, PURL, DOI, and WebCite and found $20 \%$ of the links were inaccessible in $2008 .{ }^{17}$ McMurry et al. investigated link rot in life sciences data and suggested practices for formatting links for increased persistence and approaches for versioning. ${ }^{18}$ The topic of link rot has been examined as early as 2003, in Markwell and Brooke’s “Broken Links: Just How Rapidly Do Science Education Hyperlinks Go Extinct," cited by multiple link rot studies. Ironically, this article is no longer accessible at the cited URL. ${ }^{19}$

\section{METHODOLOGY}

This study sought a set of digital objects within library institutions' digital collections websites. To locate examples of publicly accessible digital objects in digital collections, this study collected institutional websites from the Digital Library Federation's (DLF) published list of 195 members 
as of August 2019. ${ }^{20}$ Subsequent investigation aimed to find one representative digital object from unique digital collections navigable from each institution's main web page.

This study aimed to locate digital collections that met the following criteria:

1. Collections are openly available.

2. Collections are in a repository service, as opposed to highlighted content visible on an informational web page or blog.

3. Collections are gathered within a site or service that contains multiple collections, as opposed to individual digital project websites, when possible.

4. Collections are unique to an institution, as opposed to duplicated or licensed content.

These criteria were developed in an effort to find unique, publicly accessible digital objects within each institution's digital collections. To be sure, users search for and discover materials in a variety of ways and in numerous services, but studying the information-seeking behavior of users looking for digital objects or digital collections is outside the scope of this study. Ultimately, digital collections indexed by search engines or available in aggregator services like DPLA often contain links to collections and objects in their institutionally hosted platforms. Users who discover these materials are likely to be directed to the sites this study investigated.

For the purposes of this study, at least one digital collection was investigated from each DLF institution. Multiple sites for an institution were investigated when more than one publicly accessible site or service met the above criteria. When digital collections at an institution were delivered only through the library catalog discovery service, reasonable attempts were made to delimit discoverable digital collections content. In total, 183 digital collections were identified for this study.

Once digital collections were located, subsequent investigation aimed to locate individual digital objects within them. While digital objects represent diverse materials available in a variety of formats, for ease of comparing approaches between institutions, a mixture of individual digital images, multipage digital items, and audiovisual materials were examined. Objects for this study were primarily available in websites containing a variety of collections and format types with common display characteristics despite format differences, and no additional efforts were made to locate equal or proportional digital object formats at each institution. One representative digital object was identified per digital collection, totaling 183 digital objects.

Once a digital object was located at an institution, the object's unique identifier, format, persistent URL, persistent URL label, method of link resolution (if identifiable), and citation were collected with particular focus on the object's persistent URL, if available. Commonly used persistent URL types and their URL components can be identified, as seen in table 1; however, any means of persistence was collected if clearly identified. After examining initial results, the object's provided citation, if available, was added to the list of data collected since many digital collection platforms provide recommended citations for individual objects. 
Table 1. Commonly used persistent URL methods and corresponding URL components

\begin{tabular}{|l|l|}
\hline Persistent URL type & URL component \\
\hline Archival Resource Key (ARK) & ark:/ \\
\hline Digital Object Identifier (DOI) & doi.org/ (or doi:) \\
\hline Handle & hdl.handle.net \\
\hline Persistent URL (PURL) & purl. \\
\hline
\end{tabular}

\section{RESULTS}

Most institutions have a single digital collection site or service that met the selection criteria for this study. Some appear to have multiple digital collection repositories, often separated by digital object format or library department, and many institutions have collections that are only publicly accessible through discrete project web sites, such as digital exhibits or focused digital humanities research projects. Out of 195 DLF member institutions, 171 had publicly accessible digital collections. Of these 171 institutions, $153 \mathrm{had}$ digital collections services/sites that adhered to the criteria of this study, while 21 had only project-focused digital collections sites. Since several institutions had more than one digital collection platform accessible via their main institutional website, a population of 183 digital collections were investigated. One representative digital object from each collection was gathered, consisting of 107 digital images, 73 multipage items, and 3 audiovisual items (totaling 183).

Table 2. Number of instances of digital collection platforms identified

\begin{tabular}{|l|c|c|}
\hline Platform & Number & Percentage of total (183) \\
\hline Custom or unidentifiable & 53 & $29 \%$ \\
\hline CONTENTdm & 46 & $25 \%$ \\
\hline Islandora & 19 & $10 \%$ \\
\hline DSpace & 11 & $6 \%$ \\
\hline Samvera & 11 & $6 \%$ \\
\hline Omeka & 10 & $5 \%$ \\
\hline Internet Archive & 7 & $4 \%$ \\
\hline Digital Commons & 6 & $3 \%$ \\
\hline Fedora Custom & 4 & $2 \%$ \\
\hline Luna & 3 & $2 \%$ \\
\hline XTF & 3 & $2 \%$ \\
\hline Artstor & 2 & $1 \%$ \\
\hline IIIF Server & 2 & $1 \%$ \\
\hline Primo & 2 & $1 \%$ \\
\hline Aspace & 1 & $1 \%$ \\
\hline Elevator & 1 & $1 \%$ \\
\hline Knowvation & 1 & $1 \%$ \\
\hline Veridian & 1 & $1 \%$ \\
\hline
\end{tabular}


As seen in table 2, almost a third of digital collection platforms encountered appear to be customdeveloped or customized to not reveal the software platform upon which they were based. Of the platform-based services encountered where software was identifiable, 17 different platforms were used and the top five were CONTENTdm, Islandora, DSpace, Samvera (Hyrax, Avalon, Curation Concerns, etc.), and Omeka.

Table 3. Occurrence of persistent links in surveyed digital collections, method of link persistence, and persistent link labels

\begin{tabular}{|l|l|l|}
\hline Persistent links? & Number & Percentage of total (183) \\
\hline No/unknown & 93 & $51 \%$ \\
\hline Yes/ persistence claimed & 90 & $49 \%$ \\
\hline & & \\
\hline Persistent link method & Number & Percentage of total (90) \\
\hline Unknown & 33 & $37 \%$ \\
\hline Handle & 27 & $30 \%$ \\
\hline ARK & 19 & $21 \%$ \\
\hline DOI & 6 & $7 \%$ \\
\hline PURL & 5 & $6 \%$ \\
\hline & & \\
\hline Persistent link label & Number & Percentage of total (90) \\
\hline Other & 24 & $26.7 \%$ \\
\hline Permalink & 22 & $24.4 \%$ \\
\hline Identifier & 13 & $14.4 \%$ \\
\hline [No label given] & 10 & $11.1 \%$ \\
\hline Permanent Link & 7 & $7.8 \%$ \\
\hline URI & 5 & $6 \%$ \\
\hline Persistent Link & 3 & $3.3 \%$ \\
\hline Handle & 2 & $2.2 \%$ \\
\hline Link to the book & 2 & $2.2 \%$ \\
\hline Persistent URL & 2 & $2.2 \%$ \\
\hline $\begin{array}{l}\text { aTwenty-four other persistent link labels were reported, }{ }^{21} \text { each occurring } \\
\text { only once. }\end{array}$ &
\end{tabular}

As seen in table 3, the numbers of digital objects with and without publicly accessible persistent (or seemingly persistent) links were nearly equal. Among the digital objects with persistent links, the majority claimed persistence without a discernible resolution method, with the rest divided between Handle, ARK, DOI, and PURL. These objects also had 33 different labels for these links in the public-facing interface. The top five labels were: Permalink (22), Identifier (13), Permanent Link (7), URI (5), and Persistent Link (3).

As seen in table 4, the majority of digital objects surveyed had a unique item identifier in their publicly viewable item record. The majority did not offer a citation in the item's publicly viewable record. Among items that offered citations, the majority contained a link to the item, and three offered downloadable citation formats only, such as Endnote, Zotero, and Mendeley. 
Table 4. Various digital object characteristics surveyed

\begin{tabular}{|l|l|l|}
\hline Unique item identifier in item record & Number & Percentage of total (183) \\
\hline Yes & 132 & $72 \%$ \\
\hline No & 51 & $28 \%$ \\
\hline & & \\
\hline Citation in item record & Number & Percentage of total (183) \\
\hline Yes & 65 & $36 \%$ \\
\hline No & 118 & $64 \%$ \\
\hline & & \\
\hline Citations containing links to item & Number & Percentage of total (65) \\
\hline Yes & 39 & $60 \%$ \\
\hline Downloadable citation format only & 3 & $5 \%$ \\
\hline No & 23 & $35 \%$ \\
\hline
\end{tabular}

\section{DISCUSSION}

Since proper citation practice dictates choosing the URL most likely to provide continuing access to a resource, it follows that providing persistent URLs to resources such as digital objects or digital collections is also a good practice. It is encouraging to see a large number of institutions surveyed providing URLs that persist (or claim to persist). Providing persistent access to a unique digital resource implies a level of commitment to maintaining its URL into the future, requiring policies, technology, and labor resources, further augmented by costs associated with registering certain types of identifiers like DOI. ${ }^{22}$ It is likely that institutions not providing persistent (or not obviously persistent) URLs are either internally committing to preserving their objects, collections, and services through means not known to end users; are constrained by technological limitations of their digital collection platforms; hope to develop or adopt new digital library services that offer these capabilities; or lack the resources to offer persistent URLs.

The four commonly used methods of persistent link resolution-DOI, Handle, ARK, and PURLhave been used for nearly 20 years, and it is not surprising that alternative observable methods were seldom encountered in this study. Handles were the most common persistent URL method, which seems related to the digital library platform used by an institution. DSpace distributions are pre-bundled with Handle server software, for example, and 12 out of 27 platforms serving digital objects with Handles were based on DSpace (https://duraspace.org/dspace/). When choosing to implement or upgrade a digital library platform, institutions often consider several available options. Choosing a platform that offers the ability to easily create and maintain persistent URLs might be less burdensome than making URLs persist via independent or alternative means.

Thirty-three digital objects offered links that had labels implying some sort of persistence but lacked information describing the methods used or URL components consistent with commonly used methods, as seen in table 1 . To achieve persistence, there might be a combination of URL rewriting, locally implemented solutions, or nonpublic persistent URLs existing. It would benefit users, increasingly aware of the need to cite digital objects using persistent links, for digital object platforms that offer persistent linking to explicitly state that fact and ideally offer some evidence of the resolution method used. Researchers will be looking for citable persistent links that offer 
some cues signifying their persistence, whether it is clearly indicated language on the website or a URL pattern consistent with the four major methods commonly used.

The amount of variation in labeling persistent links was surprising. Commonly used digital library software platforms have default ways of labeling these fields. Nearly all of the "Reference URL" labels encountered are in CONTENTdm sites, for example. Since the concept of offering a persistent link to a digital object is not uncommon, perhaps there can be a more consistent approach to choosing the label for this content. When a researcher finds a digital object in an institutional digital library service, they might want to cite that object. Accurately citing resources in all formats is an essential research skill, and digital library platforms often try to aid users by providing dynamically generated or pre-populated citations based on unique metadata associated with that object. It was somewhat surprising to encounter these types of citation helpers that did not include persistent links. Since a digital object's preferred persistent link is often different than the URL visible in the browser, efforts should be made to make citations available containing persistent links.

There are institutions with digital collections that were not examined in this study due to a number of factors. First, this study examined the 195 institutions who were members of the Digital Library Federation, and there are 2,828 four-year postsecondary institutions in the United States as of 2018. ${ }^{23}$ Additional study could expand perceptions about persistent links for digital objects when looking beyond the DLF member institutions, which are predominantly four-year postsecondary institutions but also contain museums, public libraries, and other cultural heritage organizations.

An alternative approach to collecting this data would be to conduct user testing focused on finding and citing digital objects from a number of institutions. This approach was not used, however, since the initial goal of this study was to see how peer digital library institutions have employed persistent links and citations across a broad yet contained spectrum. As one librarian with extensive digital library experience, my approach to locating these platforms and resources is subject to subconscious bias I may have accumulated over my professional career, but I would hope that my experience makes me more able to locate these platforms and materials than the average user.

Digital library platforms are numerous, and often institutions have several of them with varying degrees of public visibility or connectivity to their institution's main library website. This study's findings for any particular institution are not as authoritative as self-reported information from the institution itself. While a survey aimed at collecting direct responses from institutions might have yielded more accuracy, a potentially low response rate would also make it difficult to truly know what methods of persistent linking peer institutions are employing, especially with the majority of these resources being openly findable and accessible. Still, further study with selfreported information could shed more light on the decisions to provide certain methods of persistent links to objects within their chosen digital collection platforms. Moreover, it is possible that some digital object formats are more likely to have persistent URLs than others. Newer formats such as three-dimensional digital objects, commonly cited resources like data sets, and scholarship held in institutional repositories could be available in digital library services similar to those surveyed in this study with different persistent URL characteristics. Additional study could aim to survey populations of digital objects by format across multiple institutions to investigate any correlation between persistent URLs and object format. 


\section{CONCLUSION}

Unique digital collections at digital library institutions are made openly accessible to the public in a variety of ways, including digital library software platforms and digital library aggregator services. Regardless of how users find these materials, best practices require users to cite URLs for these materials that are most likely to continue to provide access to them. Persistent URLs are a common way to ensure cited URLs to digital objects remain accessible. Commonly used methods of issuing and maintaining persistent URLs can be identified in digital object records within digital collection platforms available at these institutions. This study identified characteristics about these digital objects, their platforms, prevalence of persistent URLs in their records, and the way these URLs are presented to users. Findings indicate that DLF member institutions are split evenly between providing and not providing publicly discernible persistent URLs with wide variation on how these URLs are presented and explained to users. Decisions made in developing and maintaining digital collection platforms and the types of URLs made available to users impact which URLs users cite and the possibility of others encountering these resources through these citations. Embarking on this study also was prompted by digital collection migrations at Indiana University, and these findings provide us interesting examples of persistent URL usage at other institutions and ways to improve the user experience in digital collection platforms.

\section{ENDNOTES}

${ }^{1}$ The Chicago Manual of Style Online (Chicago: University of Chicago Press, 2017), ch. 14, sec. 7.

${ }^{2}$ Arthur Allison et al., "Digital Identity Matters," Journal of the American Society for Information Science \& Technology 56, no. 4 (2005): 364-72, https://doi.org/10.1002/asi.20112.

${ }^{3}$ Yuk Hui, "What Is a Digital Object?" Metaphilosophy 43, no. 4 (2012): 380-95, https://doi.org/10.1111/j.1467-9973.2012.01761.x.

${ }^{4}$ Clifford Lynch, "Authenticity and Integrity in the Digital Environment: An Exploratory Analysis of the Central Role of Trust" Council on Library and Information Resources (CLIR), 2000, https://www.clir.org/pubs/reports/pub92/lynch/.

${ }^{5}$ G. Benoit and Lisa Hussey, "Repurposing Digital Objects: Case Studies Across the Publishing Industry," Journal of the American Society for Information Science \& Technology 62, no. 2 (2011): 363-74, https://doi.org/10.1002/asi.21465.

${ }^{6}$ Angela Dappert et al., "Describing and Preserving Digital Object Environments," New Review of Information Networking 18, no. 2 (2013): 106-73, https://doi.org/10.1080/13614576.2013.842494.

${ }^{7}$ Christos Strubulis et al., "A Case Study on Propagating and Updating Provenance Information Using the CIDOC CRM," International Journal on Digital Libraries 15, no. 1 (2014): 27-51, https://doi.org/10.1007/s00799-014-0125-z.

${ }^{8}$ William Y. Arms, “Uniform Resource Names: Handles, PURLs, and Digital Object Identifiers," Communications of the ACM 44, no. 5 (2001): 68, https://doi.org/10.1145/374308.375358. 
${ }^{9}$ Lukas Koster, "Persistent Identifiers for Heritage Objects," Code4Lib Journal 47 (2020), https://journal.code4lib.org/articles/14978.

${ }^{10}$ Albert W. Simmonds, “The Digital Object Identifier (DOI)," Publishing Research Quarterly 15, no. 2 (1999): 10, https://doi.org/10.1007/s12109-999-0022-2; Norman Paskin, "Digital Object Identifiers," Information Services \& Use 22, no. 2/3 (2002): 97, https://doi.org/10.3233/ISU2002-222-309.

${ }^{11}$ David Sidman and Tom Davidson, "A Practical Guide to Automating the Digital Supply Chain with the Digital Object Identifier (DOI)," Publishing Research Quarterly 17, no. 2 (2001): 9, https://doi.org/10.1007/s12109-001-0019-y; Andy Weissberg, "The Identification of Digital Book Content," Publishing Research Quarterly 24, no.4 (2008): 255-60, https://doi.org/10.1007/s12109-008-9093-8.

12 Susanne DeRisi, Rebecca Kennison, and Nick Twyman, "The What and Whys of DOIs," PLoS Biology 1, no. 2 (2003): 133-34, https://doi.org/10.1371/journal.pbio.0000057; Diane J. Skiba, “Digital Object Identifiers: Are They Important to Me?," Nursing Education Perspectives 30, no. 6 (2009): 394-95, https://doi.org/10.1016/j.lookout.2008.06.012; Janna Neumann and Jan Brase, "DataCite and DOI names for Research Data," Journal of Computer-Aided Molecular Design 28, no. 10 (2014): 1035-41, https://doi.org/10.1007/s10822-014-9776-5.

13 John Kunze, “Towards Electronic Persistence Using ARK Identifiers," California Digital Library, 2003, https://escholarship.org/uc/item/3bg2w3vs.

${ }^{14}$ Keith E. Shafer, Stuart L. Weibel, and Erik Jul, “The PURL Project,” Journal of Library Administration 34, no. 1-2 (2001): 123, https://doi.org/10.1300/J111v34n01_19.

${ }^{15}$ Keith E. Shafer, “ARMs, OCLC Internet Services, and PURLs," Journal of Library Administration 34, no. 3-4 (2001): 385, https://doi.org/10.1300/J111v34n03 19.

${ }^{16}$ Cendi Persistent Identification Task Group, "Persistent Identification: A Key Component of an Egovernment Infrastructure," New Review of Information Networking 10, no. 1 (2004): 97-106, https://doi-org/10.1080/13614570412331312021.

17 Erick Ducut, Fang Liu, and Paul Fontelo, "An Update on Uniform Resource Locator (URL) Decay in MEDLINE Abstracts and Measures for Its Mitigation," BMC Medical Informatics \& Decision Making 8, no. 1 (2008): 1-8, https://doi.org/10.1186/1472-6947-8-23.

18 Julie A. McMurry et al., "Identifiers for the 21st Century: How to Design, Provision, and Reuse Persistent Identifiers to Maximize Utility and Impact of Life Science Data," PLoS Biology 15, no. 6 (2017): 1-18, https://doi.org/10.1371/journal.pbio.2001414.

${ }^{19}$ John Markwell and David Brooks, "Broken Links: Just How Rapidly Do Science Education Hyperlinks Go Extinct?” (2003), cited by many and previously available from: http://wwwclass.unl.edu/biochem/url/broken links.html [currently non-functional].

20 "Our Member Institutions," Digital Library Federation (2020), https://www.diglib.org/about/members/. 
${ }^{21}$ Twenty-four labels used only once: Archival Resource Key; ARK; Bookmark this page at; Citable link; Citable Link to this Page; Citable URI; Copy; Copy and Paste this URL; Digital Object URL; DOI; Identifier (HDL); Item; Link; Local Identifier; Permanent URL; Permanently Link to this Resource; Persistent Link to this Item; Persistent Link to this Record; Please use this identifier to cite or link to this item; Related Resources; Resource Identifier; Share; Share Link/Location; To cite or link to this item, use this identifier.

${ }^{22}$ One of the Frequently Asked Questions (https://www.doi.org/faq.html) states that DOI registration fees vary.

${ }^{23}$ National Center for Education Statistics, “Table 317.10. Degree-granting postsecondary institutions, by control and level of institution: Selected years, 1949-50 through 2017-18," in Digest of Education Statistics, 2018, https://nces.ed.gov/programs/digest/d18/tables/dt18 317.10.asp. 